MR Masruha, Division of Child Neurology, Department of Neurology and Neurosurgery, Botucatu Street 720, Federal University of Sao Paulo, Sao Paulo 04023-9000, Brazil).

COMMENT. This case is reported as the first example of pseudolymphoma associated with ethosuximide. Histologically, a lymphoid cell proliferation caused effacement of normal node architecture and a false appearance of malignancy. Pseudolymphoma may result from an hypersensitivity or idiosyncratic reaction, the drug acting as an antigen and triggering an immune reaction. Urticaria, Stevens-Johnson syndrome, systemic lupus erythematosus, eosinophilia, leokopenia, thrombocytopenia, aplastic anemia, and liver dysfunction have been reported with ethosuximide. Other anticonvulsants, phenytoin and lamotrigine, are reported to cause pseudolymphoma. Adverse effects of antiepileptic drugs, including idiosyncratic reactions are reviewed by Perucca $\mathrm{E}$ and Meador KJ (Arch Neurol Scand December 2005;112 (Suppl 181):30-35).

\title{
ETIOLOGY AND OUTCOME OF STATUS EPILEPTICUS
}

The etiology and outcome of status epilepticus (SE) in 135 children ( 76 boys, 59 girls), ages 1 month to 15 years, admitted to Nemazee Hospital, Shiraz, Iran, between 1999 and 2004, were studied retrospectively, and outcomes were rated according to the Glasgow Outcome Score (GOS). The mean +/- SD age was $4.14+/-3.8$ years; $44(33 \%)$ were younger than 12 months, $29(21 \%)$ were $1-3$ years old, and $62(46 \%)$ were older than 3 years. Prolonged febrile seizure was the etiology in $69(51 \%) ; 57 \%$ of FS patients were $<12$ months old, $45 \% 1-3$ years, and $50 \%>3$ years. Idiopathic SE accounted for a total of $50(37 \%)$ cases, and was associated with discontinuation of anticonvulsant drug in 38 (28\%). Symptomatic SE was the cause in $16(11.9 \%) ; 10$ due to CNS infection, 4 metabolic disorders, and 2 trauma-related. Etiology did not differ significantly between age groups $(\mathrm{P}=0.736)$ or sex $(\mathrm{P}=0.156)$.

Fourteen $(10.4 \%)$ died in hospital, and $81(60 \%)$ were discharged well. Death was related to a prolonged febrile seizure in 4, idiopathic SE in 6, and symptomatic CNS infection in 3. Patients with symptomatic SE had the worst outcome, with $63 \%$ morbidity and mortality; the morbidity and mortality rate was $48 \%$ in idiopathic SE cases, and $29 \%$ for febrile seizure SE cases. Mean +/- SD duration of hospitalization was 7+/-9.7 days (range, 168 days); it did not differ with etiology but was shorter for patients with good outcome. (Asadi-Pooya AA, Poordast A. Etiologies and outcomes of status epilepticus in children. Epilepsy Behav November 2005;7:502-505). (Dr AA Asadi-Pooya, Shiraz University of Medical Sciences, Shiraz, Iran).

COMMENT. Fever is the most common cause of status epilepticus (SE) in children, even in those older than 3 years. The etiology of SE is significantly correlated with the outcome, symptomatic cases secondary to CNS infection having the worst, and prolonged febrile seizures the most favorable outcome. Factors in outcome of SE in adolescents and adults in the Netherlands were the underlying cause, noncompliance with AED treatment, systemic infection, and duration of the SE. (Scholtes FB et al. Epilepsia 1995;35:11041112). Noncompliance with AED therapy or inadequate instruction regarding the use of rectal diazepam administered in the home explained the need for admission of children with 
SE in a study in the UK (Matthes JWA, Wallace SJ. Dev Med Child Neurol 1995;37:226231).

The risk of SE in children with an initial diagnosis of epilepsy is $9.5 \%$, according to a prospective community-based cohort study of 613 children in New York (Berg AT et al. Neurology 2004;63:1027-1034). The risk is increased in children with a previous history of $\mathrm{SE}$, in younger age groups, and in those with symptomatic etiology. The risk was $14 \%$ in symptomatic cases and $2.6 \%$ in the idiopathic group. The authors agree with the UK practice, recommending abortive therapy (rectal diazepam) in the home for patients at high risk of SE. The importance of seizure prevention and early abortive therapy is stressed, especially in symptomatic epilepsies. A clear predetermined plan of action, prompt administration of appropriate AEDs in adequate doses, and attention to apnea, hypoventilation, fever and infection, or metabolic abnormalities are outlined by the Epilepsy Foundation of America's working group on SE (Dodson WE et al. JAMA 1993;270:854-859).

\section{INFECTIOUS DISORDERS}

\section{PCR IN DIAGNOSIS OF LYME NEUROBORRELIOSIS}

A prospective study of 57 hospitalized patients with active neuroborreliosis (NB) and proved CSF antibodies was conducted using PCR for the detection of specific DNA in plasma, CSF and urine, at Faculty Hospital Bulovka; Charles University, Prague; and Hospital Nymburk, Czech Republic. Bannwarth's syndrome (lymphocytic meningoradiculitis) was present in 29 patients, acute meningoencephalitis in 5, subacute encephalitis in 3 , meningitis in 6 , polyneuritis in 9 , and facial palsy in 5 . Neurological abnormalities were mild in $25(44 \%)$, moderate in $17(30 \%)$, and severe in $15(26 \%)$. Symptoms improved with antibiotic treatment in $84 \%$, and the remainder improved after 3 months, with no relapses.

Before treatment, $36(63.1 \%)$ patients tested PCR positive in all parallel specimens, $28(49.1 \%)$ were positive in urine, $20(35 \%)$ in CSF, and $16(28 \%)$ in plasma. Immediately after treatment, $17(30 \%)$ were positive in urine, $8(14 \%)$ after 3 months, and 1 persisted positive after 6 months. The highest sensitivity of PCR was found in urine, and the lowest in plasma. The results support the use of PCR in the diagnosis of NB. (Picha D, Moravcova L, Zdarsky E et al. PCR in lyme neuroborreliosis: a prospective study. Acta Neurol Scand November 2005;112:287-292). (Respond: Dusan Picha PhD, First Clinic for Infectious Diseases, Faculty Hospital Bulovka, 18081 Prague, Czech Republic).

COMMENT. PCR testing for spirochetal DNA in urine or CSF, using appropriate primers, is a useful diagnostic tool in patients suspected of neuroborreliosis (NB).

Assessment of serum antibodies to a panel of Borrelia-specific antigens is of value in the laboratory diagnosis of NB at presentation of symptoms (Heikkila T et al. New antigens for serological diagnosis of neuroborreliosis in children. Pediatr Inf Dis J Aug 2005;24:709712). In addition to CSF lymphocytic pleocytosis and CSF antiflagella antibodies, all 7 patients with definite NB and 7 of 13 with probable NB had serum $\operatorname{IgG}$ antibodies to 2 of 3 novel antigens at presentation. 